\title{
EXISTENCE AND UNIQUENESS OF POSITIVE EIGENFUNCTIONS FOR CERTAIN EIGENVALUE SYSTEMS
}

\author{
RU-YING XUE AND YI-MIN YANG
}

\begin{abstract}
The existence and uniqueness of eigenvalues and positive eigenfunctions for some quasilinear elliptic systems are considered. Some necessary and sufficient conditions which guarantee the existence and uniqueness of eigenvalues and positive eigenfunctions are given.
\end{abstract}

\section{$\S 1$. Introduction}

Let $\Omega$ be a bounded domain in $R^{n}$ with smooth boundary $\partial \Omega$. For $p \in(1, \infty)$, we denote by $\Delta_{p}$ the $p$-Laplacian defined by $\Delta_{p} u=\operatorname{div}(\mid \nabla$ $\left.\left.u\right|^{p-2} \nabla u\right)$. It is well-known that, when $a(x) \in L^{\infty}(\Omega)$ and $\max (a(x), 0) \not \equiv 0$, the eigenvalue problem

$$
-\Delta_{p} u=\lambda a(x)|u|^{p-2} u \quad \text { in } \quad \Omega, u=0 \quad \text { on } \quad \partial \Omega
$$

has a unique eigenvalue $\lambda_{0}$ with nonnegative eigenfunctions. More precisely, the eigenvalue $\lambda_{0}$ is simple, i.e., the set of all solutions of (1.1) with $\lambda=\lambda_{0}$ consists of $\left\{t \phi_{0}: t \in \mathbf{R}^{1}\right\}$, where $\phi_{0}$ is an eigenfunction of (1.1) corresponding to $\lambda_{0}$ such that $\phi_{0} \in C^{1, \beta}(\bar{\Omega})$ for some $\beta \in(0,1)$ and $\phi_{0}(x)>0$ for all $x \in \Omega$ (see [1], [2]). In fact we have

$$
\lambda_{0}=\inf \left\{\int_{\Omega}|\nabla u|^{p} d x: u \in W_{0}^{1, p}(\Omega), \int_{\Omega} a(x)|u|^{p} d x=1\right\},
$$

and the solutions of (1.1) for $\lambda=\lambda_{0}$ are the minimizers of (1.2) (see [2]).

When we consider the existence and uniqueness of nonnegative eigenfunctions for elliptic eigenvalue systems, the following example shows that the situation is different.

ExAMPLE. Let $A(x)$ be a $L^{\infty}(\Omega)$ function satisfying $\max \{A(x), 0\} \not \equiv 0$ and $\max \{-A(x), 0\} \not \equiv 0$. For $d>2$, we denote by $k_{1}$ and $k_{2}$ two positive

Received December 19, 2000.

2000 Mathematics Subject Classification: 35J65, 35D05. 
constants satisfying $0<k_{1}<1<k_{2}$ and $k^{-1 / 2}+k^{1 / 2}=d$. Choose $C_{1}$ and $C_{2}$ such that (we assume $\alpha_{2}+\beta_{2} \neq 2$ )

$$
C_{1} k_{1}^{1-\beta_{2}}-C_{2} k_{1}^{\alpha_{2}-1}=C_{1} k_{2}^{1-\beta_{2}}-C_{2} k_{2}^{\alpha_{2}-1}=d .
$$

Let $\lambda_{0}$ and $\phi_{0}$ be the eigenvalue and the positive eigenfunction of

$$
-\Delta u=\lambda A(x) u \quad \text { in } \quad \Omega, u=0 \quad \text { on } \quad \partial \Omega .
$$

We choose $a_{1}(x)=A(x), b_{1}(x)=-A(x), a_{2}(x)=C_{2} A(x), b_{2}(x)=C_{1} A(x)$ and $\alpha_{2}+\beta_{2} \neq 2$. Then we have $\max \left\{a_{j}(x), 0\right\} \not \equiv 0$ and $\max \left\{b_{j}(x), 0\right\} \not \equiv 0$, and $\left(k_{1} \phi_{0}, \phi_{0}\right)$ and $\left(k_{2} \phi_{0}, \phi_{0}\right)$ are two positive eigenfunctions associated with eigenvalue $\lambda_{0} / d$ of the following eigenvalue system

$$
\left\{\begin{array}{l}
-\Delta u=\lambda\left[a_{1}(x) u^{1 / 2} v^{1 / 2}+a_{2}(x) u^{\alpha_{2}} v^{1-\alpha_{2}}\right] \text { in } \Omega \\
-\Delta v=\lambda\left[b_{1}(x) u^{1 / 2} v^{1 / 2}+b_{2}(x) u^{1-\beta_{2}} v^{\beta_{2}}\right] \text { in } \Omega \\
u=v=0 \text { on } \partial \Omega
\end{array}\right.
$$

In this article, we shall prove that a similar uniqueness result holds for certain elliptic eigenvalue systems with nonnegative coefficients. Consider elliptic eigenvalue systems of the form

$$
\left\{\begin{array}{l}
-\Delta_{p} u=\lambda \sum_{i=1}^{K} a_{i}(x) u^{\alpha_{i}} v^{p-1-\alpha_{i}} \text { in } \Omega \\
-\Delta_{q} v=\lambda \sum_{j=1}^{N} b_{i}(x) u^{q-1-\beta_{j}} v^{\beta_{j}} \text { in } \Omega \\
u=v=0 \text { on } \partial \Omega
\end{array}\right.
$$

where $1<p<+\infty, 1<q<+\infty, 0 \leq \alpha_{i} \leq p-1(i=1,2, \cdots, K), 0 \leq \beta_{j} \leq$ $q-1(j=1,2, \cdots, N), a_{i}(x)(i=1,2, \cdots, K)$ and $b_{j}(x)(j=1,2, \cdots, N)$ are nonnegative $L^{\infty}(\Omega)$ functions.

The main theorems obtained in this article are

TheOREM 1.1. The eigenvalue of (1.3) with nontrivial nonnegative eigenfunctions is unique. Nontrivial nonnegative eigenfunctions of (1.3) are positive.

Theorem 1.2. (1) When $\alpha_{1}<p-1$ and $\beta_{1}<q-1$, or $\alpha_{i}=p-1$ and $\beta_{j}<q-1$ for $i=1,2, \cdots, K$ and $j=1,2, \cdots, N,(1.3)$ possesses a unique eigenvalue with positive eigenfunctions, the corresponding positive eigenfunction is unique up to a scalar multiple. 
(2) When $\alpha_{i}=p-1$ for all $i=1,2, \cdots, K, \beta_{1}=q-1$ and $\beta_{j}<q-1$ for $j=2, \cdots, N,(1.3)$ has a unique eigenvalue with positive eigenfunctions if and only if

$$
\begin{aligned}
& \inf \left\{\int_{\Omega}|\nabla u|^{p} d x: \int_{\Omega} \sum_{i=1}^{K} a_{i}(x)|u|^{p} d x=1, u \in W_{0}^{1, p}(\Omega)\right\} \\
< & \inf \left\{\int_{\Omega}|\nabla v|^{q} d x: \int_{\Omega} b_{1}(x)|v|^{q} d x=1, v \in W_{0}^{1, q}(\Omega)\right\} .
\end{aligned}
$$

The corresponding positive eigenfunctions, if they exist, are unique up to a scalar multiple.

(3) When $\alpha_{i}=p-1$ and $\beta_{j}=q-1$ for $i=1,2, \cdots, K, j=1,2, \cdots, N$, (1.3) possesses an eigenvalue with positive eigenfunctions if and only if

$$
\begin{aligned}
& \inf \left\{\int_{\Omega}|\nabla u|^{p} d x: \int_{\Omega}\left[\sum_{i=1}^{K} a_{i}(x)\right]|u|^{p} d x=1, u \in W_{0}^{1, p}(\Omega)\right\} \\
= & \inf \left\{\int_{\Omega}|\nabla v|^{q} d x: \int_{\Omega}\left[\sum_{j=1}^{N} b_{j}(x)\right]|v|^{q} d x=1, v \in W_{0}^{1, q}(\Omega)\right\} .
\end{aligned}
$$

In this case, the corresponding positive eigenfunctions, if they exist, are not unique up to a scalar multiple.

By a positive eigenfunction of the eigenvalue system (1.3) corresponding to an eigenvalue $\lambda$ we mean a weak solution $(u, v) \in W_{0}^{1, p}(\Omega) \times W_{0}^{1, q}(\Omega)$ satisfying $u, v \in L^{\infty}(\Omega)$ and $u>0, v>0$ in $\Omega$. A nonnegative eigenfunction of (1.3) is a pair $(u, v) \in W_{0}^{1, p}(\Omega) \times W_{0}^{1, q}(\Omega)$, which does not vanish identically in $\Omega$ and satisfies $u, v \in L^{\infty}(\Omega)$ and $u \geq 0, v \geq 0$ in $\Omega$. A nontrivial nonnegative eigenfunction is a nonnegative eigenfunction of (1.3), each component of which does not vanish identically in $\Omega$.

This article is organized as follows. In section 2 we recall some wellknown results for the single equation with $\Delta_{p}$. That (1.3) has a unique eigenvalue with nontrivial nonnegative eigenfunctions (Theorem 1.1) and that the positive eigenfunctions are unique up to a scalar multiple are proved in Section 3. In Section 4 we consider the existence of branches of nonnegative (or positive) solutions for some quasilinear elliptic systems. The existence of positive eigenfunctions (Theorem 1.2) is considered in section 5 . 
In this article, we shall write $\left(u_{1}, v_{1}\right) \geq\left(u_{2}, v_{2}\right)$ if $u_{1} \geq u_{2}$ and $v_{1} \geq v_{2}$, $\left(u_{1}, v_{1}\right)>\left(u_{2}, v_{2}\right)$ if $u_{1}>u_{2}$ and $v_{1}>v_{2}$. We also denote by $\|(u, v)\|=$ $\sup _{x \in \Omega}[|u(x)|+|v(x)|]$.

\section{§2. Some results for a single equation with $\Delta_{p}$}

In this section we recall some well-known results for the single equation with $\Delta_{p}$. Consider the following Dirichlet problem

$$
-\Delta_{p} u=f(x) \quad \text { in } \quad \Omega, \quad u=0 \quad \text { on } \quad \partial \Omega
$$

where $p>1, f(x) \in L^{\infty}(\Omega)$ with the norm $\|f\| \stackrel{\text { def. }}{=} \sup _{x \in \Omega}|f|$. By the variational method we know that the Dirichlet problem (2.4) has a unique solution $u \in W_{0}^{1, p}(\Omega)$. We first introduce the weak comparison principle, which follows from the same argument as that used in Lemma 4.1 in [3] and the fact that $\left[\psi_{p}(\vec{x})-\psi_{p}(\vec{y})\right] \cdot(\vec{x}-\vec{y})=0$ implies $\vec{x}=\vec{y}$.

Lemma 2.1. Assume that $u_{1}, u_{2} \in W^{1, p}(\Omega)$, respectively, are weak solutions of

$$
\begin{aligned}
& -\Delta_{p} u_{1}=f_{1}(x) \quad \text { in } \quad \Omega, \quad u=g_{1} \quad \text { on } \quad \partial \Omega, \\
& -\Delta_{p} u_{2}=f_{2}(x) \quad \text { in } \quad \Omega, \quad u=g_{2} \quad \text { on } \quad \partial \Omega,
\end{aligned}
$$

with $f_{1} \leq f_{2}$ in $L^{q}(\Omega)$ and $g_{1} \leq g_{2}$ in $W^{\frac{1}{q}, p}(\partial \Omega)$, where $q=\frac{p}{p-1}$. Then $u_{1} \leq u_{2}$ almost everywhere in $\Omega$.

Choose $R_{0}$ so large that $\bar{\Omega} \subset\left\{x:|x|<R_{0}\right\}$. Clearly,

$$
0 \leq U(|x|)=n^{\frac{1}{1-p}}\left(R_{0}^{\frac{p}{p-1}}-|x|^{\frac{p}{p-1}}\right) \in W^{1, p}\left(|x|<R_{0}\right)
$$

is a positive radial weak solution of the equation

$$
-\Delta_{p} U=1 \quad \text { for } \quad|x| \leq R_{0}, \quad U=0 \quad \text { on } \quad|x|=R_{0}
$$

The following lemma comes from Lemma 2.1.

LEMmA 2.2. Assume that there exists a constant $M$ such that $0 \leq$ $f(x) \leq M^{p-1}$ in $\Omega$. Then the weak solution $u$ of $(2.4)$ satisfies $0 \leq u(x) \leq$ $M U(|x|)$ almost everywhere in $\Omega$. 
The following strong comparison principle comes from [4].

Lemma 2.3. ([4]) Assume $u_{1}, u_{2} \in W_{0}^{1, p}(\Omega)$, respectively, are weak solution of (2.5) and (2.6) with $g_{1}=g_{2}=0, f_{1}, f_{2} \in L^{\infty}(\Omega)$ satisfying $0 \leq f_{1} \leq f_{2}$ in $\Omega$. Then either $u_{1} \equiv u_{2} \geq 0$ in $\Omega$ or else

$$
0 \leq u_{1}<u_{2} \quad \text { in } \quad \Omega, \quad 0 \geq \frac{\partial u_{1}}{\partial \nu}>\frac{\partial u_{2}}{\partial \nu} \quad \text { on } \quad \partial \Omega,
$$

where $\vec{\nu}$ denotes the outward unit normal vector at $\partial \Omega$.

Denote by $C(\bar{\Omega})$ the space of all continuous functions defined on $\bar{\Omega}$ with the standard norm

$$
\|u\| \stackrel{\text { def. }}{=} \sup _{x \in \Omega}|u(x)|
$$

and let $V_{+}$be the positive cone in $C(\bar{\Omega}), V_{+}=\{u \in C(\bar{\Omega}), u \geq 0$ in $\Omega\}$. Let $f(x, u, v), g(x, u, v)$ be nonnegative functions defined in $\Omega \times[0,+\infty) \times$ $[0,+\infty)$ and satisfy, for any fixed positive constant $M, f, g \in L^{\infty}(\Omega \times$ $[0, M] \times[0, M])$. For any $(u, v) \in V_{+} \times V_{+}$, by variational methods there exists a unique weak solution $(\bar{u}, \bar{v}) \in W_{0}^{1, p}(\Omega) \times W_{0}^{1, q}(\Omega)$ satisfying

$$
\left\{\begin{array}{l}
-\Delta_{p} \bar{u}=f(x, u, v) \text { in } \Omega, \\
-\Delta_{q} \bar{v}=g(x, u, v) \text { in } \Omega, \\
\bar{u}=\bar{v}=0 \text { on } \partial \Omega
\end{array}\right.
$$

Lemma 2.2 and the regularity result proved in [5] mean there exists a constant $\beta \in(0,1)$ depending solely upon $p, q$ and $n$, and another constant $C$ depending solely upon $p, q, n,\|f(x, u, v)\|$ and $\|g(x, u, v)\|$, such that $\bar{u}, \bar{v} \in C_{0}^{1, \beta}(\bar{\Omega})$ with the Hölder norm $\|\bar{u}\|_{1+\beta, \bar{\Omega}} \leq C$ and $\|\bar{v}\|_{1+\beta, \bar{\Omega}} \leq C$. Define a mapping

$$
T:(u, v) \longmapsto(\bar{u}, \bar{v})=T(u, v) .
$$

Obviously, $T$ is a self-mapping of $V_{+} \times V_{+}$, and $T: V_{+} \times V_{+} \rightarrow V_{+} \times V_{+}$is continuous and relatively compact.

\section{$\S 3 . \quad$ Uniqueness of eigenvalues and eigenfunctions}

In this section, we consider the uniqueness of eigenvalues with nontrivial nonnegative eigenfunctions of (1.3). We first prove the uniqueness of eigenvalues associated with nontrivial nonnegative eigenfunctions. 
Proof of Theorem 1.1. Suppose that $\left(u_{1}, v_{1}\right),\left(u_{2}, v_{2}\right)$ are two nontrivial nonnegative eigenfunctions associated with eigenvalues $\Lambda_{1}, \Lambda_{2}$, respectively, for the eigenvalue system (1.3). Lemma 2.1, Lemma 2.3 and the regularity result in [5] imply that $\Lambda_{1}, \Lambda_{2}>0 ; u_{j}, v_{j} \in C^{1, \beta}(\bar{\Omega}), j=1,2$, are positive in $\Omega$; and

$$
\frac{\partial u_{1}}{\partial \nu}<0, \frac{\partial u_{2}}{\partial \nu}<0, \frac{\partial v_{1}}{\partial \nu}<0 \text { and } \frac{\partial v_{2}}{\partial \nu}<0 \quad \text { on } \partial \Omega
$$

Thus, without loss of generality, we may assume that $(0,0)<\left(u_{1}, v_{1}\right) \leq$ $\left(u_{2}, v_{2}\right)$ and $\Lambda_{1} \leq \Lambda_{2}$. Let us consider the following elliptic system:

$$
\left\{\begin{array}{l}
-\Delta_{p} u=\Lambda_{1} \sum_{i=1}^{K} a_{i}(x) u^{\alpha_{i}} v^{p-1-\alpha_{i}}+\left(\Lambda_{2}-\Lambda_{1}\right) \sum_{i=1}^{K} a_{i}(x) u_{2}^{\alpha_{i} v_{2}^{p-1-\alpha_{i}}} \text { in } \Omega, \\
-\Delta_{q} v=\Lambda_{1} \sum_{j=1}^{N} b_{j}(x) u^{q-1-\beta_{j}} v^{\beta_{j}}+\left(\Lambda_{2}-\Lambda_{1}\right) \sum_{j=1}^{N} b_{j}(x) u_{2}^{q-1-\beta_{j}} v_{2}^{\beta_{j}} \text { in } \Omega, \\
u=v=0 \text { on } \partial \Omega .
\end{array}\right.
$$

Observe that $\left(u_{2}, v_{2}\right)$ is a solution of (3.11). By Lemma 2.1, we have $\left(\xi u_{1}, \xi v_{1} \leq T\left(\xi u_{1}, \xi v_{1}\right)\right.$ for all $\xi \in(0,+\infty)$ and $T\left(\xi u_{2}, \xi v_{2}\right) \leq\left(\xi u_{2}, \xi v_{2}\right)$ for all $\xi \in(1,+\infty)$, where $T$ is the mapping from $V_{+} \times V_{+}$to $V_{+} \times V_{+}$ defined in (2.9) for

$$
\begin{aligned}
& f(x, u, v)=\Lambda_{1} \sum_{i=1}^{K} a_{i}(x) u^{\alpha_{i}} v^{p-1-\alpha_{i}}+\left(\Lambda_{2}-\Lambda_{1}\right) \sum_{i=1}^{K} a_{i}(x) u_{2}^{\alpha_{i}} v_{2}^{p-1-\alpha_{i}}, \\
& g(x, u, v)=\Lambda_{1} \sum_{j=1}^{N} b_{j}(x) u^{q-1-\beta_{j}} v^{\beta_{j}}+\left(\Lambda_{2}-\Lambda_{1}\right) \sum_{j=1}^{N} b_{j}(x) u_{2}^{q-1-\beta_{j}} v_{2}^{\beta_{j}} .
\end{aligned}
$$

Making use of (3.10) and the fact that $(0,0)<\left(u_{1}, v_{1}\right) \leq\left(u_{2}, v_{2}\right)$, we can pick $\xi>1$ so large that $\left(u_{2}, v_{2}\right) \leq \xi\left(u_{1}, v_{1}\right)$. Then, from Lemma 2.1 we arrive at

$$
\begin{aligned}
\left(u_{2}, v_{2}\right) & \leq \xi\left(u_{1}, v_{1}\right) \leq T\left(\xi u_{1}, \xi v_{1}\right) \leq \ldots \leq T^{k}\left(\xi u_{1}, \xi v_{1}\right) \\
& \leq T^{k}\left(\xi u_{2}, \xi v_{2}\right) \leq \ldots \leq T\left(\xi u_{2}, \xi v_{2}\right) \leq \xi\left(u_{2}, v_{2}\right)
\end{aligned}
$$

The compactness of $T$ implies $T^{k}\left(\xi u_{2}, \xi v_{2}\right) \rightarrow\left(u_{3}, v_{3}\right)$ in $V_{+}$as $k \rightarrow+\infty$ for some $\left(u_{3}, v_{3}\right) \in V_{+} \times V_{+}$and

$$
\left(u_{2}, v_{2}\right) \leq\left(\xi u_{1}, \xi v_{1}\right) \leq T\left(u_{3}, v_{3}\right)=\left(u_{3}, v_{3}\right) \leq \xi\left(u_{2}, v_{2}\right)
$$


Hence $\left(u_{3}, v_{3}\right)$ is a positive solution of $(3.11)$. We claim that $\left(u_{3}, v_{3}\right)=$ $\left(u_{2}, v_{2}\right)$. If this is the case, (3.13) implies that $\left(u_{2}, v_{2}\right)=\xi\left(u_{1}, v_{1}\right)$, and hence $\Lambda_{1}=\Lambda_{2}$ as desired.

On the contrary, suppose $\left(u_{3}, v_{3}\right) \not \equiv\left(u_{2}, v_{2}\right)$. We assume that $\left(u_{3}, v_{3}\right) \leq$ $\left(u_{2}, v_{2}\right)$ is false. Consequently, by $(3.10)$ we can pick $t \in(1,+\infty)$ which is the smallest number satisfying

$$
t^{-1}\left(u_{3}, v_{3}\right) \leq\left(u_{2}, v_{2}\right)
$$

We have

$$
\begin{aligned}
t^{1-p} f\left(u_{3}, v_{3}\right) & \leq f\left(t^{-1} u_{3}, t^{-1} v_{3}\right) \\
& \leq f\left(u_{2}, v_{2}\right), t^{1-p} f\left(u_{3}, v_{3}\right) \not \equiv f\left(t^{-1} u_{3}, t^{-1} v_{3}\right)
\end{aligned}
$$

and

$$
\left\{\begin{array}{l}
-\Delta_{p}\left(t^{-1} u_{3}\right)=t^{1-p} f\left(u_{3}, v_{3}\right) \quad \text { in } \Omega \\
-\Delta_{p}\left(u_{2}\right)=f\left(u_{2}, v_{2}\right) \quad \text { in } \Omega \\
u_{3}=u_{2}=0 \quad \text { on } \partial \Omega
\end{array}\right.
$$

By (3.16) and (3.16), Lemma 2.3 implies

$$
0<t^{-1} u_{3}<u_{2} \text { in } \Omega \text {, and } \frac{\partial u_{2}}{\partial \nu}<\frac{\partial\left(t^{-1} u_{3}\right)}{\partial \nu}<0 \text { on } \partial \Omega .
$$

Then we find $\bar{t} \in(1, t)$ such that

$$
0<\bar{t}^{-1} u_{3} \leq u_{2} \text { in } \Omega
$$

a contradiction to our choice of $t$. That nontrivial nonnegative eigenfunctions of (1.3) are positive follows from Lemma 2.3 directly.

Remark 3.1. It is obvious that (1.3) may have a unique eigenvalue for which the eigenfunctions are of the form $(u, 0)$ with $u>0$, and a unique eigenvalue for which the eigenfunctions are of the form $(0, v)$ with $v>0$. If (1.3) has an eigenvalue with nontrivial nonnegative eigenfunctions, we know that the eigenvalue is unique by Theorem A. Hence, (1.3) has at most three eigenvalues associated with nonnegative eigenfunctions. It is possible that (1.3) may have no eigenvalues with nontrivial nonnegative (positive) eigenfunctions. 
TheOREM 3.1. Except the case that $\alpha_{i}=p-1(i=1,2, \cdots, K)$ and $\beta_{j}=q-1(j=1,2, \cdots, N)$, positive eigenfunctions of the eigenvalue problem (1.3) are unique up to scalar multiples.

Proof. By Theorem 1.1, an eigenvalue of (1.3) with positive eigenfunctions is positive and unique. Assume that $\left(\phi_{1}, \psi_{1}\right)$ and $\left(\phi_{2}, \psi_{2}\right)$ are two positive eigenfunctions of (1.3), associated with an eigenvalues $\lambda$. It is sufficient to prove that $\left(\phi_{1}, \psi_{1}\right)$ and $\left(\phi_{2}, \psi_{2}\right)$ are colinear. By Lemma 2.3 ,

$$
\begin{aligned}
& \phi_{i}(x)>0, \quad \psi_{i}(x)>0 \quad \text { for } \quad x \in \Omega, i=1,2, \\
& \frac{\partial \phi_{i}}{\partial \nu}<0, \quad \frac{\partial \psi_{i}}{\partial \nu}<0, \quad \text { for } \quad x \in \partial \Omega, i=1,2 .
\end{aligned}
$$

Thus, we can choose two positive numbers $C_{1}<C_{2}$ such that

$$
C_{1}\left(\phi_{1}, \psi_{1}\right) \leq\left(\phi_{2}, \psi_{2}\right) \leq C_{2}\left(\phi_{1}, \psi_{1}\right)
$$

Without loss of generality, we assume that $C_{1}=1$ and $\left(\phi_{1}, \psi_{1}\right) \leq\left(\phi_{2}, \psi_{2}\right)$. We claim that $\left(\phi_{1}, \psi_{1}\right)$ and $\left(\phi_{2}, \psi_{2}\right)$ are linearly dependent. Indeed, otherwise there exists the smallest number $t_{0}>1$ such that

$$
t_{0}\left(\phi_{1}, \psi_{1}\right) \geq\left(\phi_{2}, \psi_{2}\right), \quad t_{0}\left(\phi_{1}, \psi_{1}\right) \not \equiv\left(\phi_{2}, \psi_{2}\right)
$$

Let

$$
\begin{aligned}
& F(x, u, v)=\lambda \sum_{i=1}^{K} a_{i}(x) u^{\alpha_{i}} v^{p-1-\alpha_{i}} \\
& G(x, u, v)=\lambda \sum_{j=1}^{N} b_{j}(x) u^{q-1-\beta_{j}} v^{\beta_{j}} .
\end{aligned}
$$

They satisfy

$$
F\left(x, t_{0} \phi_{1}, t_{0} \psi_{1}\right) \geq F\left(x, \phi_{2}, \psi_{2}\right), \quad G\left(x, t_{0} \phi_{1}, t_{0} \psi_{1}\right) \geq G\left(x, \phi_{2}, \psi_{2}\right) .
$$

We claim that

(3.18) $F\left(x, t_{0} \phi_{1}, t_{0} \psi_{1}\right) \not \equiv F\left(x, \phi_{2}, \psi_{2}\right), G\left(x, t_{0} \phi_{1}, t_{0} \psi_{1}\right) \not \equiv G\left(x, \phi_{2}, \psi_{2}\right)$.

In fact, if

$$
F\left(x, t_{0} \phi_{1}, t_{0} \psi_{1}\right) \equiv F\left(x, \phi_{2}, \psi_{2}\right)
$$


we deduce from (3.10) that $t_{0} \phi_{1}=\phi_{2}$. It is obvious that $t_{0} \psi_{1}$ and $\psi_{2}$ are two positive solutions of the following elliptic problem

$$
-\Delta_{q} v=G\left(x, \phi_{2}, v\right) \quad x \in \Omega, \quad v=0 \quad x \in \partial \Omega
$$

When $\beta_{j}<q-1$ for some $j \in\{1,2, \cdots, N\}$, the same argument as that in the proof of Theorem 2.1 in [3] shows that (3.19) possesses at most one positive solution. Thus, we have $t_{0} \psi_{1}=\psi_{2}$, hence $\left(t_{0} \phi_{1}, t_{0} \psi_{1}\right)=\left(\phi_{2}, \psi_{2}\right)$, which is impossible because of (3.17). When $\beta_{j}=q-1$ for all $j=1,2, \cdots, N$, the uniquness of nonnegative eigenfunctions for the following eigenvalue problem

$$
-\Delta_{q} v=\lambda\left[\sum_{j=1}^{N} b_{j}(x)\right] v^{q-1} \quad x \in \Omega, \quad v=0 \quad x \in \partial \Omega
$$

shows that $t_{0} \psi_{1}$ and $\psi_{2}$ are linearly dependent, and then there exists $C_{0}$ such that $t_{0} \psi_{1}=C_{0} \psi_{2}$. But in this case we must have $\alpha_{i}<p-1$ for some $i \in$ $\{1,2, \cdots, K\}$, so we obtain $C_{0}=1$ from the hypothesis $F\left(x, t_{0} \phi_{1}, t_{0} \psi_{1}\right) \equiv$ $F\left(x, \phi_{2}, \psi_{2}\right)$. Thus $\left(t_{0} \phi_{1}, t_{0} \psi_{1}\right)=\left(\phi_{2}, \psi_{2}\right)$, which is impossible because of (3.17). Hence (3.18) holds.

By Lemma 2.2 and the regularity result of Lieberman [5],

$$
\left(\phi_{i}(x), \psi_{i}(x)\right) \in C^{1, \beta}(\bar{\Omega}) \times C^{1, \beta}(\bar{\Omega}), \quad i=1,2
$$

with some $\beta \in(0,1)$. A combination of (3.17) and (3.18) with Lemma 2.3 yields that

$$
\begin{aligned}
& t_{0}\left(\phi_{1}, \psi_{1}\right)>\left(\phi_{2}, \psi_{2}\right) \quad x \in \Omega \\
& \left(\frac{\partial\left(t_{0} \phi_{1}\right)}{\partial \nu}, \frac{\partial\left(t_{0} \psi_{1}\right)}{\partial \nu}\right)<\left(\frac{\partial \phi_{2}}{\partial \nu}, \frac{\partial \psi_{2}}{\partial \nu}\right)<(0,0) \quad x \in \partial \Omega .
\end{aligned}
$$

Hence, there exists a positive number $t_{1} \in\left(0, t_{0}\right)$ such that

$$
t_{1}\left(\phi_{1}, \psi_{1}\right) \geq\left(\phi_{2}, \psi_{2}\right)
$$

a contradiction to our choice of the number $t_{0}$. Hence $\left(\phi_{1}, \psi_{1}\right)$ and $\left(\phi_{2}, \psi_{2}\right)$ are linearly dependent. 


\section{§4. Bifurcation properties of nonnegative solutions}

In this section, we consider the bifurcation properties of nonnegative solutions of the following elliptic system

$$
\begin{cases}-\Delta_{p} u=\lambda \sum_{i=1}^{K} a_{i}(x) u^{\alpha_{i}} v^{p-1-\alpha_{i}}+f(x, u, v, \lambda), & x \in \Omega \\ -\Delta_{q} v=\lambda \sum_{j=1}^{N} b_{j}(x) u^{q-1-\beta_{j}} v^{\beta_{j}}+g(x, u, v, \lambda), & x \in \Omega \\ u=v=0, \quad x \in \partial \Omega & \end{cases}
$$

We assume that

$(F)$. For any given $M>0, f(x, u, v, \lambda)$ and $g(x, u, v, \lambda)$ are two nonnegative $L^{\infty}$ functions defined in $\Omega \times[0, M] \times[0, M] \times[0, M], f(x, u, v, 0)=0$, $g(x, u, v, 0)=0$, and

$$
\lim _{t \rightarrow 0^{+}} f(x, t u, t v, \lambda) t^{1-p}=0, \quad \lim _{t \rightarrow 0^{+}} g(x, t u, t v, \lambda) t^{1-q}=0,
$$

uniformly with respect to $(x, u, v) \in \Omega \times(0,1] \times(0,1]$ and $\lambda$ on bounded intervals.

Theorem 4.1. Assume $f(x, u, v, \lambda)$ and $g(x, u, v, \lambda)$ satisfy $(F)$. If $\left(\lambda_{0}, 0,0\right)$ is a bifurcation point of nonnegative nontrivial solutions for (4.21), then $\lambda_{0}$ is an eigenvalue of (1.3) with nonnegative eigenfunctions.

Proof. Let

$$
\begin{aligned}
& F(x, u, v, \lambda)=\lambda \sum_{i=1}^{K} a_{i}(x) u^{\alpha_{i}} v^{p-1-\alpha_{i}}+f(x, u, v, \lambda), \\
& G(x, u, v, \lambda)=\lambda \sum_{j=1}^{N} b_{j}(x) u^{q-1-\beta_{j}} v^{\beta_{j}}+g(x, u, v, \lambda) .
\end{aligned}
$$

For a function $h \in L^{\infty}(\Omega)$, we denote by $u$ the weak solution of the following elliptic boundary value problem

$$
-\Delta_{p} u=h(x), \quad x \in \Omega, \quad u=0 \quad x \in \partial \Omega .
$$

Let $T_{p}$ be the mapping defined by $T_{p}(h(x))=u(x)$. Lemma 2.1, Lemma 2.2 and the regularity results of Lieberman [5] imply $T_{p}: V_{+} \rightarrow V_{+}$is a completely continuous mapping. Let $J$ be the mapping defined by

$$
J[\lambda, u, v]=\left[T_{p}(F(x, u, v, \lambda)), T_{q}(G(x, u, v, \lambda))\right] .
$$


and let $\left\{\left(\lambda_{k}, u_{k}, v_{k}\right)\right\}$ be a sequence of nonnegative nontrivial solutions of (4.21) satisfying $\lim _{k \rightarrow \infty} \lambda_{k}=\lambda_{0}, \lim _{k \rightarrow \infty}\left(u_{k}, v_{k}\right)=(0,0)$ in $V_{+} \times V_{+}$and

$$
\left(u_{k}, v_{k}\right)=J\left[\lambda_{k}, u_{k}, v_{k}\right]
$$

Denote $t_{k}=\left\|\left(u_{k}, v_{k}\right)\right\|$. Let $\left(\bar{u}_{k}, \bar{v}_{k}\right)=t_{k}^{-1}\left(u_{k}, v_{k}\right)$. It follows from (4.22) that

$$
\left(\bar{u}_{k}, \bar{v}_{k}\right)=\left(T_{p}\left[t_{k}^{1-p} F\left(x, t_{k} \bar{u}_{k}, t_{k} \bar{v}_{k}, \lambda_{k}\right)\right], T_{q}\left[t_{k}^{1-q} G\left(x, t_{k} \bar{u}_{k}, t_{k} \bar{v}_{k}, \lambda_{k}\right)\right]\right)
$$

Notice that $\left\|\left(\bar{u}_{k}, \bar{v}_{k}\right)\right\|=1$ and $t_{k} \rightarrow 0^{+}$as $k \rightarrow \infty$. For any $\epsilon>0$, the hypothesis $(F)$ shows that there exists $k_{0}$ so large that

$$
\left|t_{k}^{1-p} f\left(x, t_{k} \bar{u}_{k}, t_{k} \bar{v}_{k}, \lambda_{k}\right)\right|<\epsilon,\left|t_{k}^{1-q} g\left(x, t_{k} \bar{u}_{k}, t_{k} \bar{v}_{k}, \lambda_{k}\right)\right|<\epsilon,
$$

as $k \geq k_{0}$. (4.23) and $\left\|\left(\bar{u}_{k}, \bar{v}_{k}\right)\right\|=1$ imply that $F\left(x, t_{k} \bar{u}_{k}, t_{k} \bar{v}_{k}, \lambda_{k}\right)$ and $G\left(x, t_{k} \bar{u}_{k}, t_{k} \bar{v}_{k}, \lambda_{k}\right)$ are bounded sequences in $V_{+}$. It follows from the regularity results of Lieberman[5] that $\left\{\left(\bar{u}_{k}, \bar{v}_{k}\right)\right\}$ is a bounded sequence in the Banach space $C_{0}^{1, \beta}(\bar{\Omega})$ for some positive constant $\beta$. Thus there exist $\left(u_{0}, v_{0}\right) \in C_{0}^{1}(\bar{\Omega}) \times C_{0}^{1}(\bar{\Omega})$ and a subsequence ( still denoted by $\left.\left\{\left(u_{k}, v_{k}\right)\right\}\right)$ such that

$$
\lim _{k \rightarrow \infty}\left(\bar{u}_{k}, \bar{v}_{k}\right)=\left(u_{0}, v_{0}\right) \quad \text { in } \quad C_{0}^{1}(\bar{\Omega}) \times C_{0}^{1}(\bar{\Omega}),
$$

and $\left\|\left(u_{0}, v_{0}\right)\right\|=\lim _{k \rightarrow \infty}\left\|\left(\bar{u}_{k}, \bar{v}_{k}\right)\right\|=1$. Combining (4.24) with (4.25) yields

$$
\begin{aligned}
& \lim _{k \rightarrow \infty}\left(t_{k}^{1-p} F\left(x, t_{k} \bar{u}_{k}, t_{k} \bar{v}_{k}, \lambda_{k}\right), t_{k}^{1-q} G\left(x, t_{k} \bar{u}_{k}, t_{k} \bar{v}_{k}, \lambda_{k}\right)\right) \\
= & \left(\lambda_{0} \sum_{i=1}^{K} a_{i}(x) u_{0}^{\alpha_{i}} v_{0}^{p-1-\alpha_{i}}, \lambda_{0} \sum_{j=1}^{N} b_{j}(x) u_{0}^{q-1-\beta_{j}} v_{0}^{\beta_{j}}\right) \text { in } \quad V_{+} \times V_{+} .
\end{aligned}
$$

Since $T_{p}$ and $T_{q}$ are completely continuous operators mapping $V_{+}$into itself, it follows from (4.23) and (4.27) that

$$
\left(u_{0}, v_{0}\right)=\left(T_{p}\left[\lambda_{0} \sum_{i=1}^{K} a_{i}(x) u_{0}^{\alpha_{i}} v_{0}^{p-1-\alpha_{i}}\right], T_{q}\left[\lambda_{0} \sum_{j=1}^{N} b_{j}(x) u_{0}^{q-1-\beta_{j}} v_{0}^{\beta_{j}}\right]\right)
$$

with $\left\|\left(u_{0}, v_{0}\right)\right\|=1$. Hence $\lambda_{0}$ is an eigenvalue of (1.3) with a nonnegative eigenfunction $\left(u_{0}, v_{0}\right)$. We complete the proof of Theorem 4.1. 
For $x \in \Omega$, we denote $\frac{1}{d(x)}=\inf \{|x-y|: y \in \partial \Omega\}$. Let $\left\{\left(\lambda_{k}, u_{k}, v_{k}\right)\right\}$ be a branch of nonnegative solutions of (4.21) such that $\lim _{k \rightarrow \infty} \lambda_{k}=\lambda_{0}$ and $\lim _{k \rightarrow \infty}\left(u_{k}, v_{k}\right)=(0,0)$ in $V_{+} \times V_{+}$. Denote by $\left(\bar{u}_{k}, \bar{v}_{k}\right)=\left\|\left(u_{k}, v_{k}\right)\right\|^{-1}\left(u_{k}, v_{k}\right)$. From the proof of Theorem 4.1 we know that

$$
\lim _{k \rightarrow \infty}\left(\bar{u}_{k}, \bar{v}_{k}\right)=\left(u_{0}, v_{0}\right) \quad \text { in } \quad C_{0}^{1}(\bar{\Omega}) \times C_{0}^{1}(\bar{\Omega})
$$

and that $\lambda_{0}$ is an eigenvalue of (1.3) with a nonnegative eigenfunction $\left(u_{0}, v_{0}\right)$ satisfying $\left\|\left(u_{0}, v_{0}\right)\right\|=1$. (4.27) implies

$$
\lim _{k \rightarrow \infty}\left(d(x) \bar{u}_{k}, d(x) \bar{v}_{k}\right)=\left(d(x) u_{0}, d(x) v_{0}\right) \quad \text { in } \quad V_{+} \times V_{+} .
$$

When $\left(u_{0}, v_{0}\right)$ is a nontrivial nonnegative eigenfunction of (1.3) and when there exists some $i \in\{1,2, \cdots, K\}$ (or $j \in\{1,2, \cdots, N\}$ ) such that $\alpha_{i}<p-1$ ( or $\beta_{j}<q-1$ ), Theorem 1.1, Theorem 3.1 and Lemma 2.3 mean that there exists a positive constant $\delta>0$, which is independent of $\left\{\left(\lambda_{k}, u_{k}, v_{k}\right)\right\}$, such that $\inf \left\{d(x) u_{0}(x) \mid x \in \Omega\right\} \geq \delta$ and $\inf \left\{d(x) v_{0}(x) \mid x \in\right.$ $\Omega\} \geq \delta$. By (4.28) we can choose two positive constant $C_{1}<C_{2}$ satisfying

$$
\begin{aligned}
C_{1} & \leq \frac{\delta-\left|d(x)\left(u_{k}(x)-u_{0}(x)\right)\right|}{\left|d(x) v_{0}(x)\right|+\left|d(x)\left(v_{k}(x)-v_{0}(x)\right)\right|} \leq \frac{u_{k}(x)}{v_{k}(x)} \\
& =\frac{d(x) u_{k}(x)}{d(x) v_{k}(x)} \leq \frac{\left|d(x) u_{0}(x)\right|+\left|d(x)\left(u_{k}(x)-u_{0}(x)\right)\right|}{\delta-\left|d(x)\left(v_{k}(x)-v_{0}(x)\right)\right|} \leq C_{2}
\end{aligned}
$$

for $k$ large enough.

When $\left(u_{0}, v_{0}\right)$ is a nonnegative eigenfunction of $(1.3)$ with $v_{0} \equiv 0$, it is obvious that nonnegative eigenfunctions of (1.3) of the form $(u, 0)$ and $\|(u, 0)\|=1$ are unique. As above we get

$$
\frac{v_{k}(x)}{u_{k}(x)}=\frac{d(x) v_{k}(x)}{d(x) u_{k}(x)} \leq \frac{\left|d(x) v_{k}(x)\right|}{\delta-\left|d(x)\left(u_{k}(x)-u_{0}(x)\right)\right|}
$$

for $k$ large enough, which shows that $v_{k}(x)=o\left(u_{k}(x)\right)$ uniformly for $x \in \Omega$ as $k \rightarrow \infty$. Thus, we have

Remark 4.1. Assume that $\left(\lambda_{0}, 0,0\right)$ is a bifurcation point of (4.21) and that $\left\{\left(\lambda_{k}, u_{k}, v_{k}\right)\right\}$ is a branch of nonnegative solutions bifurcating from $\left(\lambda_{0}, 0,0\right)$. Then,

(1). If $\lambda_{0}$ is an eigenvalue of (1.3) with nonnegative eigenfunctions of the form $(u, 0)$, then $\lim _{k \rightarrow \infty} \frac{v_{k}(x)}{u_{k}(x)}=0$ uniformly for $x \in \Omega$. 
(2). If $\lambda_{0}$ is an eigenvalue of (1.3) with nonnegative eigenfunctions of the form $(0, v)$, then $\lim _{k \rightarrow \infty} \frac{u_{k}(x)}{v_{k}(x)}=0$ uniformly for $x \in \Omega$.

(3). If $\lambda_{0}$ is an eigenvalue of (1.3) with nontrivial nonnegative eigenfunctions and there exists some $i \in\{1,2, \cdots, K\}$ (or $j \in\{1,2, \cdots, N\}$ ) such that $\alpha_{i}<p-1$ ( or $\beta_{j}<q-1$ ), then there exist positive numbers $C_{1} \leq C_{2}$, which are independent of the sequence $\left\{\left(\lambda_{k}, u_{k}, v_{k}\right)\right\}_{k=1}^{\infty}$, such that $C_{1} v_{k}(x) \leq u_{k}(x) \leq C_{2} v_{k}(x)$ for $x \in \Omega$ and $k$ large enough.

In the sequel, we shall consider the existence of a branch of nonnegative solutions of (4.21). Denote by $P_{\epsilon}=\left\{(u, v) \in V_{+} \times V_{+}:\|u\|+\|v\| \leq \epsilon\right\}$. Using an argument similar to that in proof of Lemma 2.4 of [7], we have

LEMmA 4.2. Assume the functions $f(x, u, v, \lambda)$ and $g(x, u, v, \lambda)$ satisfy $(F)$. Suppose that there is a positive number $\bar{\lambda}$ such that, for $\lambda>\bar{\lambda},(\lambda, 0,0)$ is not a bifurcation point for $(4.21)$ and $\operatorname{deg}\left(I-J[\lambda, \cdot, \cdot], P_{\epsilon},(0,0)\right)=0$ for $\epsilon$ small enough. Then there exists $\lambda_{0} \in(0, \bar{\lambda}]$ such that the set of nontrivial nonnegative solutions of (4.21) contains an unbounded subcontinuum bifurcating from $\left(\lambda_{0}, 0,0\right)$, where $J$ is a completely continuous mapping defined in the proof of Theorem 4.1.

Let $\Lambda_{1}$ be the eigenvalue of the problem

$$
-\Delta_{p} u=\lambda a_{1}(x)|u|^{p-2} u \quad \text { in } \quad \Omega, u=0 \quad \text { on } \quad \partial \Omega
$$

with the positive eigenfunction $\phi(x)$ satisfying $\|\phi\|=1$. Let $\Lambda_{2}$ be the eigenvalue of the problem

$$
-\Delta_{q} v=\lambda b_{1}(x)|v|^{q-2} v \quad \text { in } \quad \Omega, v=0 \quad \text { on } \quad \partial \Omega
$$

with the positive eigenfunction $\psi(x)$ satisfying $\|\psi\|=1$. It is obvious that $\phi, \psi \in C^{1, \beta}(\bar{\Omega})$ and that there exist two positive constant $C_{1} \leq C_{2}$ satisfying $0<C_{1} \psi(x) \leq \phi(x) \leq C_{2} \psi(x)$ for all $x \in \Omega$. Let

$\Lambda_{3}=\max \{\lambda: \lambda$ is an eigenvalue of (1.3) with nonnegative eigenfunctions $\}$

$$
\Lambda=\max \left\{\Lambda_{1} C_{2}^{p-1-\alpha_{1}}, \Lambda_{2} C_{1}^{\beta_{1}+1-q}, \Lambda_{3}\right\}
$$

By Remark 3.1 we deduce that $0<\Lambda<\infty$.

LEmma 4.3. Let $\lambda>\Lambda$. For any $\epsilon>0$ small we have $\operatorname{deg}(I-$ $\left.J[\lambda, \cdot, \cdot], P_{\epsilon},(0,0)\right)=0$. 
Proof. Define

$$
\begin{aligned}
F_{t}(x, u, v) & =\lambda \sum_{i=1}^{K} a_{i}(x) u^{\alpha_{i}} v^{p-1-\alpha_{i}}+t f(x, u, v, \lambda), \\
G_{t}(x, u, v) & =\lambda \sum_{j=1}^{N} b_{j}(x) u^{q-1-\beta_{j}} v^{\beta_{j}}+\operatorname{tg}(x, u, v, \lambda) . \\
H[t, u, v] & =\left(T_{p}\left[F_{t}(x, u, v)\right], T_{q}\left[G_{t}(x, u, v)\right]\right) .
\end{aligned}
$$

Clearly, $H:[0,1] \times V_{+} \times V_{+} \rightarrow V_{+} \times V_{+}$is a completely continuous mapping. We claim that the operator equation $(u, v)-H[t, u, v]=(0,0)$ has no solution on $\left\{(u, v) \in V_{+} \times V_{+},\|u\|+\|v\|=\epsilon\right\}$ for $t \in[0,1]$ and $\epsilon$ small. Indeed, otherwise there exist $\left\{\left(u_{k}, v_{k}\right)\right\}$ and $\left\{t_{k}\right\}$ such that $(0,0) \not \equiv\left(u_{k}, v_{k}\right) \rightarrow(0,0)$ in $V_{+} \times V_{+}, t_{k} \rightarrow t_{0} \in[0,1]$ and $\left(u_{k}, v_{k}\right)=H\left[t_{k}, u_{k}, v_{k}\right]$. Using the same argument as that in the proof of Theorem 4.1, we can prove that $\lambda$ is an eigenvalue of (1.3) with nonnegative eigenfunctions. Thus $\lambda \leq \Lambda$, which is impossible. We have

$$
\begin{aligned}
\operatorname{deg}\left(I-J[\lambda, \cdot, \cdot], P_{\epsilon},(0,0)\right) & =\operatorname{deg}\left(I-H[1, \cdot, \cdot], P_{\epsilon},(0,0)\right) \\
& =\operatorname{deg}\left(I-H[0, \cdot, \cdot], P_{\epsilon},(0,0)\right)
\end{aligned}
$$

Define

$$
\begin{aligned}
A_{t}(x, u, v) & =\lambda \sum_{i=1}^{K} a_{i}(x) u^{\alpha_{i}} v^{p-1-\alpha_{i}}+t, \\
B_{t}(x, u, v) & =\lambda \sum_{i=1}^{N} b_{j}(x) u^{q-1-\beta_{j}} v^{\beta_{j}}+t, \\
S[t, u, v] & =\left(T_{p}\left[A_{t}(x, u, v)\right], T_{q}\left[B_{t}(x, u, v)\right]\right) .
\end{aligned}
$$

Clearly, $S:[0,1] \times V_{+} \times V_{+} \rightarrow V_{+} \times V_{+}$is a completely continuous mapping too. The choice of $\lambda$ means that $(u, v)-S[0, u, v]=(0,0)$ has no solution on $\{(u, v) \in V \times V,\|u\|+\|v\|=\epsilon\}$ for $\epsilon$ small. We claim that $(u, v)-S[t, u, v]=$ $(0,0)$ has no solution on $P_{\epsilon}$ for $t \in(0,1]$ and $\epsilon$ small. Indeed, otherwise there exist $t_{0} \in(0,1]$ and $\left(u_{0}, v_{0}\right) \in P_{\epsilon}$ such that $\left(u_{0}, v_{0}\right)=S\left[t_{0}, u_{0}, v_{0}\right]$. Lemma 2.3 shows

$$
\left(u_{0}, v_{0}\right)>(0,0) \quad x \in \Omega, \quad\left(\frac{\partial u_{0}}{\partial \nu}, \frac{\partial v_{0}}{\partial \nu}\right)<(0,0) \quad x \in \partial \Omega .
$$

Moreover, $\left(u_{0}, v_{0}\right)$ is a supersolution of (1.3). The fact that $\lambda>\Lambda \geq$ $\Lambda_{1} C_{2}^{p-1-\alpha_{1}}$ and $\lambda>\Lambda \geq \Lambda_{2} C_{1}^{\beta_{1}+1-q}$ means that $(\phi, \psi)$ is a subsolution 
of (1.3). Choose $\delta>0$ so small that $\delta(\phi, \psi) \leq\left(u_{0}, v_{0}\right)$ for all $x \in \Omega$. Thus, $\delta(\phi, \psi)$ is a subsolution of (1.3), and by the supersolution-subsolution method, (1.3) possesses a nonnegative solution $(\bar{u}, \bar{v})$ satisfying $\delta(\phi, \psi) \leq$ $(\bar{u}, \bar{v}) \leq\left(u_{0}, v_{0}\right)$ for all $x \in \Omega$. Hence, $\lambda$ is an eigenvalue of (1.3) with a nonnegative eigenfunction $(\bar{u}, \bar{v})$. This is contrary to $\lambda>\Lambda$. Hence $(u, v)-S[t, u, v]=(0,0)$ has no solution on $P_{\epsilon}$ for $t \in(0,1]$ and $\epsilon$ small, by $(4.30)$

$$
\begin{aligned}
\operatorname{deg}\left(I-J[\lambda, \cdot, \cdot], P_{\epsilon},(0,0)\right) & =\operatorname{deg}\left(I-H[0, \cdot, \cdot], P_{\epsilon},(0,0)\right) \\
= & \operatorname{deg}\left(I-S[0, \cdot, \cdot], P_{\epsilon},(0,0)\right)=\operatorname{deg}\left(I-S[1, \cdot, \cdot], P_{\epsilon},(0,0)\right)=0 .
\end{aligned}
$$

A combination of Theorem 4.1 with Lemma 4.2 and Lemma 4.3 yields

Theorem 4.4. Assume $f(x, u, v, \lambda)$ and $g(x, u, v, \lambda)$ satisfy $(\mathrm{F})$. Then (4.21) contains an unbounded component of nonnegative solutions bifurcating from $\left(\lambda_{0}, 0,0\right)$, where $\lambda_{0}$ is one of eigenvalues of (1.3) associated with nonnegative eigenfunctions.

\section{§5. The existence of positive eigenfunctions}

In this section we shall consider the existence of positive eigenfunctions for the elliptic eigenvalue system (1.3).

Proof of Theorem 1.2. (1). Consider the following elliptic system

$$
\begin{cases}-\Delta_{p} u=\lambda \sum_{i=1}^{K} a_{i}(x) u^{\alpha_{i}} v^{p-1-\alpha_{i}}+\lambda v^{p+1}, & x \in \Omega, \\ -\Delta_{q} v=\lambda \sum_{j=1}^{N} b_{j}(x) u^{q-1-\beta_{j}} v^{\beta_{j}}+\lambda u^{q+1}, & x \in \Omega, \\ u=v=0 . \quad x \in \partial \Omega . & \end{cases}
$$

By Theorem 4.4 and Lemma 2.3 the system (5.31) possesses a sequence of positive solutions $\left\{\left(u_{k}, v_{k}, \lambda_{k}\right)\right\}$ such that $\lim _{k \rightarrow \infty} \lambda_{k}=\lambda_{0}, \lim _{k \rightarrow \infty}\left(u_{k}, v_{k}\right)=$ $(0,0)$ in $V_{+} \times V_{+}$, and $\lambda_{0}$ is an eigenvalue of (1.3) with nonnegative eigenfunctions. Thus, $\lambda_{0}>0$. What we want to prove is to show that $\lambda_{0}$ is an eigenvalue associated with nontrivial nonnegative eigenfunctions of (1.3). Denote by

$$
\begin{aligned}
& B_{k}(x)=\sum_{i=1}^{K} a_{i}(x)\left(\frac{v_{k}}{u_{k}}\right)^{p-1-\alpha_{i}}+v_{k}^{p+1} u_{k}^{1-p}, \\
& C_{k}(x)=\sum_{j=1}^{N} b_{j}(x)\left(\frac{u_{k}}{v_{k}}\right)^{q-1-\beta_{j}}+u_{k}^{q+1} v_{k}^{1-q} .
\end{aligned}
$$


By Lemma $2.3, B_{k}(x), C_{k}(x) \in L^{\infty}(\Omega)$ and satisfy

$$
-\Delta_{p} u_{k}=\lambda_{k} B_{k}(x) u_{k}^{p-1} \quad x \in \Omega, \quad u_{k}=0 \quad x \in \partial \Omega
$$

and

$$
-\Delta_{q} v_{k}=\lambda_{k} C_{k}(x) v_{k}^{q-1} \quad x \in \Omega, \quad v_{k}=0 \quad x \in \partial \Omega .
$$

The uniqueness of eigenvalues with nonnegative eigenfunctions for a signal equation shows (for example see [2]) that

$$
\begin{aligned}
& \lambda_{k}=\inf \left\{\frac{\int_{\Omega}|\nabla u|^{p} d x}{\int_{\Omega} B_{k}(x)|u|^{p} d x}, \quad u \in W_{0}^{1, p}(\Omega)\right\}, \\
& \lambda_{k}=\inf \left\{\frac{\int_{\Omega}|\nabla v|^{q} d x}{\int_{\Omega} C_{k}(x)|v|^{q} d x}, \quad v \in W_{0}^{1, q}(\Omega)\right\} .
\end{aligned}
$$

We claim that $\lambda_{0}$ is an eigenvalue of (1.3) with nontrivial nonnegative eigenfunctions. Otherwise, Remark 4.1 shows

$$
\lim _{k \rightarrow \infty} \frac{u_{k}}{v_{k}}=0 \quad \text { or } \quad \lim _{k \rightarrow \infty} \frac{v_{k}}{u_{k}}=0 \quad \text { uniformly for } x \in \Omega,
$$

hence, for any $\epsilon>0$

$$
\frac{u_{k}}{v_{k}} \leq \epsilon \quad \text { or } \quad \frac{v_{k}}{u_{k}} \leq \epsilon \quad \text { uniformly for } x \in \Omega \text { and for } k \text { large enough. }
$$

When $\alpha_{1}<p-1$ and $\beta_{1}<q-1$, without loss of generality, we consider the case

$$
\frac{v_{k}}{u_{k}} \leq \epsilon \quad \text { uniformly for } x \in \Omega \text { and } k \text { large enough. }
$$

We deduce from the definition of $B_{k}$ and $C_{k}$

$$
B_{k} \leq 1+\sum_{i=1}^{K} a_{i}(x) \text { and } \quad C_{k} \geq b_{1}(x) \epsilon^{\beta_{1}-q+1}
$$

for all $x \in \Omega$ and $k$ large enough. Thus we get

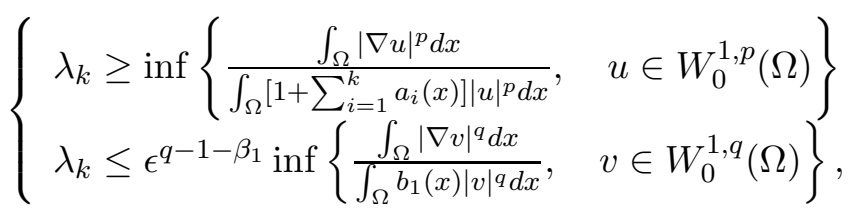


which is impossible for $\epsilon$ small enough and $k$ large.

When $\alpha_{i}=p-1$ and $\beta_{j}<q-1$ for $i=1,2, \cdots, K$ and $j=1,2, \cdots, N$ (we assume $\beta_{j} \leq \beta_{1}$ ), we have

$$
B_{k}(x) \leq 1+\sum_{i=1}^{K} a_{i}(x), \quad C_{k}(x) \geq b_{1}(x) \epsilon^{\beta_{1}-q+1}
$$

or

$$
B_{k}(x) \geq \sum_{i=1}^{K} a_{i}(x), \quad C_{k}(x) \leq \epsilon^{q-1-\beta_{1}}\left(1+\sum_{j=1}^{N} b_{j}(x)\right)
$$

for $x \in \Omega$ and $k$ large enough. We deduce that

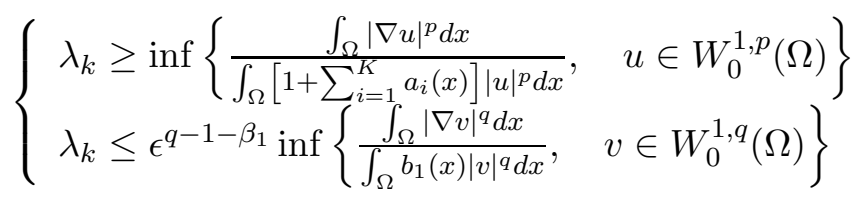

or

$$
\left\{\begin{array}{l}
\lambda_{k} \leq \inf \left\{\frac{\int_{\Omega}|\nabla u|^{p} d x}{\int_{\Omega} \sum_{i=1}^{K} a_{i}(x)|u|^{p} d x}, \quad u \in W_{0}^{1, p}(\Omega)\right\} \\
\lambda_{k} \geq \epsilon^{\beta_{1}-q+1} \inf \left\{\frac{\int_{\Omega}|\nabla v|^{q} d x}{\int_{\Omega}\left[1+\sum_{j=1}^{N} b_{j}(x)\right]|v|^{q} d x}, \quad v \in W_{0}^{1, q}(\Omega)\right\},
\end{array}\right.
$$

which is impossible when $k$ large enough and $\epsilon$ small enough. Hence we prove that $\lambda_{0}$ is an eigenvalue of (1.3) with nontrivial nonnegative eigenfunctions, and by Lemma $2.3 \lambda_{0}$ is an eigenvalue with positive eigenfunctions. The uniqueness of eigenvalues with positive eigenfunctions and the uniqueness of positive eigenfunctions of (1.3) follow from Theorem 3.1 and Theorem 1.1.

(2). Suppose that (1.3) possesses an eigenvalue $\lambda_{0}$ with a positive eigenfunction $(\phi, \psi)$. It is obvious that $\lambda_{0}>0$ and $\lambda_{0}$ is an eigenvalue with positive eigenfunctions of the following eigenvalue problems

$$
-\Delta_{p} u=\lambda\left[\sum_{i=1}^{K} a_{i}(x)\right] u^{p-1} \quad x \in \Omega, \quad u=0 \quad x \in \partial \Omega
$$

and

$(5.35)-\Delta_{q} v=\lambda\left[\sum_{j=1}^{N} b_{j}(x) \phi^{q-1-\beta_{j}} \psi^{\beta_{j}+1-q}\right] v^{q-1} \quad x \in \Omega, \quad v=0 \quad x \in \partial \Omega$. 
The uniqueness of eigenvalues with positive eigenfunctions for (5.34) or (5.35)(see [2]) shows

$$
\begin{aligned}
\lambda_{0} & =\inf \left\{\int_{\Omega}|\nabla u|^{p} d x: \int_{\Omega} \sum_{i=1}^{K} a_{i}(x)|u|^{p} d x=1, u \in W_{0}^{1, p}(\Omega)\right\} \\
& =\inf \left\{\int_{\Omega}|\nabla v|^{q} d x: \int_{\Omega}\left[\sum_{j=1}^{N} b_{j}(x) \psi^{\beta_{j}+1-q} \phi^{q-1-\beta_{j}}\right] v^{q} d x=1, v \in W_{0}^{1, q}(\Omega)\right\} \\
& <\inf \left\{\int_{\Omega}|\nabla v|^{q} d x: \int_{\Omega} b_{1}(x)|v|^{q} d x=1, v \in W_{0}^{1, q}(\Omega)\right\} .
\end{aligned}
$$

Now we prove that (1.3) possesses positive eigenfunctions. Denote by $\lambda_{0}>0$ the unique eigenvalue of (5.34) with positive eigenfunctions, and let $\phi(x) \in$ $C^{1, \beta}(\bar{\Omega}) \subset L^{\infty}(\Omega)$ be the positive eigenfunction associated to $\lambda_{0}$ satisfying $\|\phi(x)\|=1$. By the existence results in [2], the following problem

$$
\left\{\begin{array}{l}
-\Delta_{q} v=\lambda b_{2}(x) \phi^{q-1-\beta_{2}} v^{q-1} \quad x \in \Omega, \\
v=0 \quad x \in \partial \Omega
\end{array}\right.
$$

has a unique eigenvalue $\lambda_{1}>0$ associated with positive eigenfunctions. Let us denote by $\psi_{1}(x)$ the positive eigenfunction of (5.36) satisfying $\left\|\psi_{1}\right\|=1$.

On the other hand, the condition implies

$$
\lambda_{0}<\inf \left\{\int_{\Omega}|\nabla v|^{q} d x: \int_{\Omega} b_{1}(x) v^{q} d x=1, v \in W_{0}^{1, q}(\Omega)\right\},
$$

and hence we can choose a positive constant $b_{0}$ so small that

$$
\lambda_{0}<\inf \left\{\int_{\Omega}|\nabla v|^{q} d x: \quad \int_{\Omega}\left[b_{1}(x)+b_{0}\right] v^{q} d x=1, v \in W_{0}^{1, q}(\Omega)\right\} .
$$

Then the following eigenvalue problem

$$
\left\{\begin{array}{l}
-\Delta_{q} v=\lambda\left[b_{1}(x)+b_{0}\right] v^{q-1} \quad x \in \Omega \\
v=0 \quad x \in \partial \Omega
\end{array}\right.
$$

has an eigenvalue, $\lambda_{2}>\lambda_{0}$, associated with positive eigenfunctions. We denote by $\psi_{2}(x)$ a positive eigenfunction of (5.37) associated to $\lambda_{2}$ satisfying $\left|\psi_{2}(x)\right|=1$. By Lemma 2.3,

$$
\phi(x)>0, \psi_{1}(x)>0, \psi_{2}(x)>0 \quad \text { for } \quad x \in \Omega,
$$




$$
\frac{\partial \phi}{\partial \nu}<0, \frac{\partial \psi_{1}}{\partial \nu}<0, \frac{\partial \psi_{2}}{\partial \nu}<0 \text { for } \in \partial \Omega,
$$

and hence we can choose two positive constants $C_{1}$ and $C_{2}$ such that

$$
\psi_{1}(x) \leq C_{1} \phi(x), \quad \psi_{2}(x) \geq C_{2} \phi(x) \quad \text { for } \quad x \in \Omega .
$$

Choose $M>0$ large enough and $\epsilon>0$ small enough such that

$$
\begin{gathered}
\lambda_{2} b_{0} \geq \lambda_{0} \sum_{j=2}^{N} b_{j}(x)\left(C_{2} M\right)^{\beta_{j}+1-q}, \quad \lambda_{1} \epsilon^{q-1-\beta_{2}} \psi_{1}^{q-1-\beta_{2}} \leq \lambda_{0}, \\
M \psi_{2}(x) \geq \epsilon \psi_{1}(x) \quad \text { for } \quad x \in \Omega .
\end{gathered}
$$

Then $\epsilon \psi_{1}(x)$ and $M \psi_{2}(x)$ are a subsolution and a supersolution, respectively, of the following elliptic problem

$$
\left\{\begin{array}{l}
-\Delta_{q} v=\lambda_{0} \sum_{j=1}^{N} b_{j}(x) \phi^{q-1-\beta_{j}} v^{\beta_{j}} \quad \text { for } \quad x \in \Omega \\
v=0 \text { for } \in \partial \Omega
\end{array}\right.
$$

The subsolution-supersolution method shows (5.38) possess a positive solution $\psi(x)$. Obviously, $\lambda_{0}$ and $(\phi, \psi)$ are the eigenvalue and the positive eigenfunction of (1.3), respectively. The uniqueness of the eigenvalue with positive eigenfunctions and the uniqueness of positive eigenfunctions of (1.3) follows from Theorem 3.1 and Theorem 1.1.

(3). The result is obvious, we omit its proof.

\section{REFERENCES}

[1] A. Anane, Simplicitéet isolation de la premiére valeur propre du p-laplacien avec poids, C. R. Acad. Sci. Paris Ser. I Math., 305 (1987), no. 16, 725-728. (MR: 89e:35124).

[2] M. Otani and T. Teshima, On the first eigenvalue of some quasilinear elliptic equations, Proc. Japan Acad. Ser. A Math. Sci., 64 (1988), no. 1, 8-10. (MR: 89h:35257).

[3] J. Feleckinger-Pelle and P.Takac, Uniqueness of positive solutions for nonlinear co-operative systems with the p-Laplacian, Indiana Univ. Math. J., 43(4) (1994), $1227-1253$.

[4] M.Cuesta and P.Takac, A strong comparison principle for the Dirichlet p-Laplacian, Lecture Notes in Pure and Applied Mathematics, Vol.194, 79-87.

[5] G. Lieberman, Boundary regularity for solutions of degenerate elliptic equations, Nonlin. Anal. T. M. A., 12(11) (1988), 1203-1219.

[6] J. L. Vazquez, A strong maximum principle for some quasilinear elliptic equations, Appl. Math. Optim., 12 (1984), 191-202. 
[7] Y. X. Huang, Positive solutions of quasilinear elliptic equations, Research Reports in Math., 10 (1997), Stockholm Univ., Sweden.

[8] H. Amann, Fixed point equations and nonlinear eigenvalue problems in ordered Banach spaces, SIAM Rev., 18 (1978), 620-708.

[9] P. Felmer, F. R. Manasevich and F. de Thelin, Existence and uniqueness of positive solutions for certain quasilinear elliptic systems, Comm. in P. D. E., 17(11-12) (1992), 2013-2029.

[10] R. Xue and Y. Qin, Existence and uniqueness of positive eigenvalues for certain eigenvalue system, System Science and Mathematical Science, 12 (1999), 175-184.

[11] P. Lindqvist, On the equation $\operatorname{div}\left(|\nabla u|^{p-2} \nabla u\right)+\lambda|u|^{p-2} u=0$, Proc. Amer. Math. Soc., 109 (1990), 157-164.

[12] P. A. Binding and Y. X. Huang, Existence and nonexistence of positive eigenfunctions for the p-Laplacian, Proc. Amer. Math. Soc., 123(6) (1995), 1833-1838.

Ru-ying Xue

Department of Mathematics, XiXi Campus

Zhejiang University

Hangzhou 310028, Zhejiang

P. R. China

xuery@mail.hz.zj.cn

Yi-min Yang

Department of Mathematics

Hangzhou Normal College

Hangzhou 310003, Zhejiang

P. R. China 